QIAOCHU CHEN, B.Eng. ${ }^{1}$

(Corresponding author)

E-mail: chenqiaochu@hotmail.com

YAN BAI, Ph.D. ${ }^{1}$

E-mail: ybai@bjtu.edu.cn

NAN ZHENG, M.Eng. ${ }^{2}$

E-mail: 1142495548@qq.com

${ }^{1}$ Beijing Jiaotong University

No.3 Shangyuancun, Haidian District, Beijing

P. R. China

${ }^{2}$ China Railway Engineering Consulting Group Co., LTD.

No.15 Guang'an Road, Fengtai District, Beijing

P.R. China
Science in Traffic and Transport Original Scientific Paper Submitted: 20 July 2019 Accepted: 19 Feb. 2020

\title{
SIMULATION AND OPTIMIZATION OF PEDESTRIAN REGULAR EVACUATION IN COMPREHENSIVE RAIL TRANSIT HUB - A CASE STUDY IN BEIJING
}

\begin{abstract}
Extensive efforts have been made in pedestrian evacuation of urban rail transit systems, since there has emerged an increasing number of congestion problems. However, few studies focus on the comprehensive urban rail transit hubs. As a comprehensive interchange hub integrating urban railway and intercity railway lines, Beijing West Railway Station was taken as a case study object. The pedestrian evacuation characteristics were analysed first. Then, a social force-based simulation model of Beijing West Railway Station was constructed in PTV Viswalk. The model was applied to visually display a real evacuation process and help identify evacuation bottlenecks. The results showed that the risk points at different facilities had various causes and features. Furthermore, the simulation model could also be used to evaluate the effectiveness of different optimization measures as long as certain model parameters were changed beforehand.
\end{abstract}

\section{KEY WORDS}

rail transit; comprehensive transportation hub; pedestrian evacuation simulation; PTV Viswalk; optimization measures;

\section{INTRODUCTION}

In the past few decades, more comprehensive rail transportation hubs have been planned and built to improve the pedestrian travel efficiency. However, most hubs have various traffic problems due to the complexity of transfer processes. Analysing the characteristics of pedestrian evacuation in these hubs are vital for detecting the bottlenecks of evacuation capacity, improving the evacuation efficiency, and ensuring pedestrian safety.

In the recent years, the research of pedestrian evacuation in traffic hubs has been developing towards a multidisciplinary mode. Li Jin et al. [1] built a path-based network flow control model to optimize the total evacuation time. After introducing the concept of $\mathrm{K}$-shortest path, they obtained satisfactory evacuation routes and the shortest evacuation time based on the user optimal equilibrium (UE) theory of a pedestrian flow. W. J. Yu et al. [2] proposed a centrifugal force model based on pedestrian behavioural simulation and pedestrian dynamics to study the pedestrian traffic characteristics. In addition, some scholars analysed the path selection behaviour of pedestrians inside a large building [3], and then constructed a dispersive pedestrian microscopic simulation model $[4,5]$.

In order to reproduce the actual process of pedestrian evacuation in a better way, the researchers abstracted the pedestrian characteristics by physical and mathematical methods, and established various simulated evacuation models of pedestrian flow. Representative models include the cellular automaton model [6], the lattice gas model [7,8], as well as the social force model [9].

The goal of this work is to study the evacuation characteristics of pedestrians in a comprehensive rail transit hub, including behavioural characteristics and 
traffic characteristics. By constructing a simulation model of pedestrian evacuation in the hub, the bottlenecks of the evacuation can be identified and advisable optimization measures proposed.

The methodology of our study includes three steps: analysis of pedestrian evacuation characteristics in the station, the construction of the simulation model, and the simulation results analysis and optimization, which are outlined in Figure 1.

The first step is aimed at analysing pedestrian evacuation characteristics by field survey methods, including the behaviours at ticket gates, behaviours of stairs/escalator selection, and the factors affecting pedestrian walking speed.

In the second step, based on the processed field survey data in the first step, a pedestrian evacuation simulation model is constructed in PTV Viswalk (social force-based). The parameters of the simulation model are calibrated. Then the evaluation indicators for the analysis of the simulation result need to be selected.

Finally, the bottlenecks of pedestrian evacuation can be identified by analysing the simulation results. The causes and characteristics of those bottlenecks need to be analysed and the corresponding optimization measures need to be proposed. Then, the optimized simulation model is ran again to evaluate the optimization effect.

\section{FIELD SURVEY - BEIJING WEST RAILWAY STATION}

\subsection{Introduction and data processing}

Beijing West Railway Station is a typical largescale comprehensive interchange hub, which not only includes ordinary railway trains and highspeed railway trains, but also serves as an interchange station of Beijing Subway Line 7 and Line 9. Therefore, it was selected as our study object. The station contains two underground floors: B1 is an integrated transfer hall, and B2 is the platform floor of Subway Line 7 and Line 9.

To analyse the pedestrian evacuation characteristics and help construct the simulation model, a field survey was conducted at the station during the evening peak hours (5 p.m.-7 p.m.) of February 26, 2019 (Tuesday), which is the busiest time period of the station in general during a whole day. Apart from a large number of commuters entering, the intercity railway passenger flow also reaches its second peak of the day: 22 general-speed trains and 18 high-speed trains arriving during this period.

The pedestrian walking speed refers to the velocity at which the pedestrians move in a certain direction. In the video observation area, the time when the pedestrian enters and leaves the observation area is recorded. Then the time difference between the two is the pedestrian average transit time through

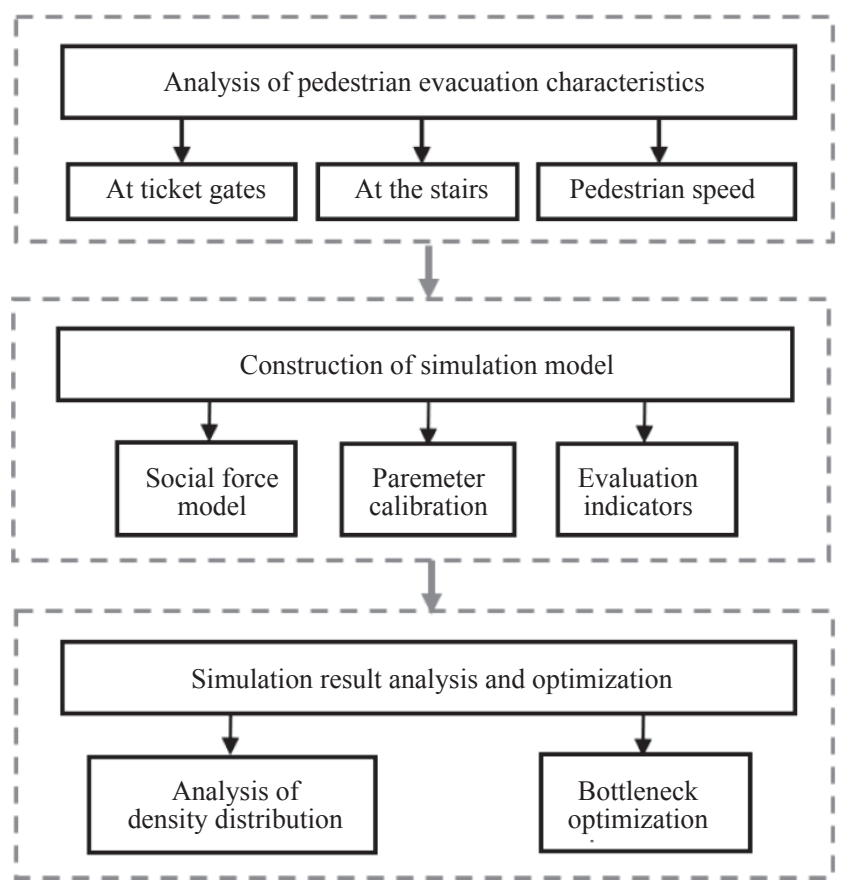

Figure 1-Methodology 
the area. Finally, the velocity is the division of the actual length of the observation area by the average transit time. Also, pedestrians are tracked to obtain their walking speed in some non-linear areas.

\subsection{Analysis of pedestrian evacuation characteristics}

\subsubsection{Factors affecting pedestrian walking speed}

\section{Gender}

There were 1,500 pedestrians taken from the video of different positions as research samples which consists of 851 males and 649 females. By analysing and processing the data samples, the ranges of pedestrian walking speeds of both genders at different positions are shown in Table 1. The results show that the walking speed of males was generally higher than that of females, which may be attributed to the height difference.

Table 1 - Expected speed distribution of pedestrians of different genders $(\mathrm{m} / \mathrm{s})$

\begin{tabular}{||l|c|c||}
\hline \multicolumn{1}{|c|}{ Expected speed } & Male & Female \\
\hline \hline Platform & $1.06-1.31$ & $0.99-1.24$ \\
\hline Transfer hall & $1.25-1.50$ & $1.20-1.45$ \\
\hline Upward stairs & $0.69-0.84$ & $0.55-0.80$ \\
\hline Downward stairs & $0.86-1.11$ & $0.77-1.02$ \\
\hline
\end{tabular}

Age

Age is also an important factor affecting pedestrian walking speed. According to the latest international classification of age groups, this paper divides passengers in the hub into four categories. The statistical data are shown in Figures 2 and 3.

Among those data samples, middle-aged and young people make up the majority of the total, whereas aged people and children make up less than $3 \%$. At the platform floor and the transfer hall, the difference of walking speed among different ages is quite obvious, while the difference is smaller on the

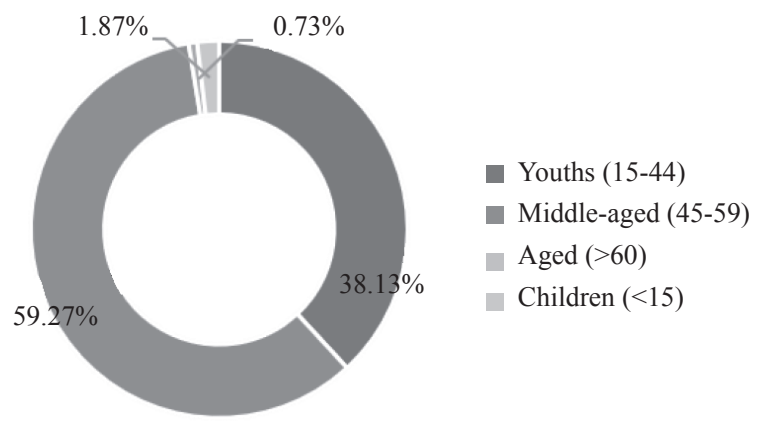

Figure 2 - Proportion of different ages

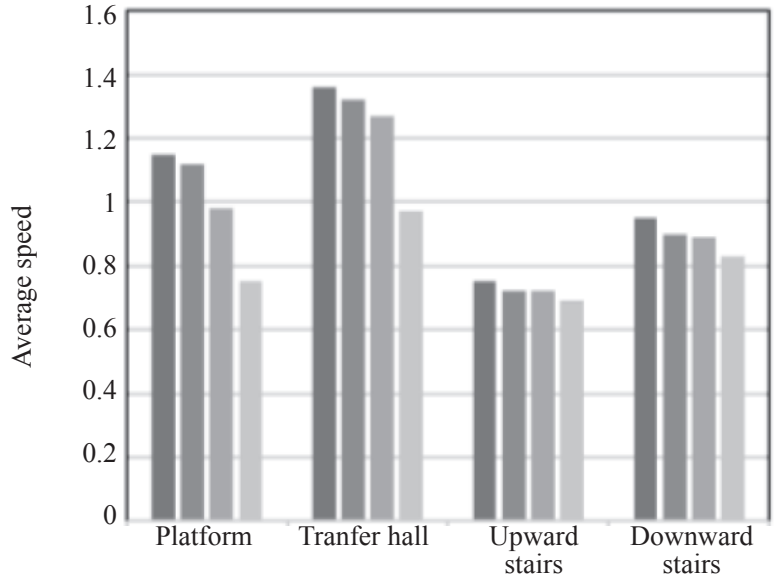

Figure 3 - Average speed of different ages

stairs. In addition, the average walking speed of middle-aged and young people at different facilities are relatively close.

\section{Load condition}

In this paper, the pedestrians carrying large luggage or backpacks are included in the load group, while the pedestrians who carry small packets are excluded. The obtained data were processed and the results are shown in Figure 4.

According to the results, the load condition has a significant impact on the walking speed of the pedestrians: the maximal speed of loaded people is only $81 \%$ of the unloaded ones and the minimum is only $63 \%$.

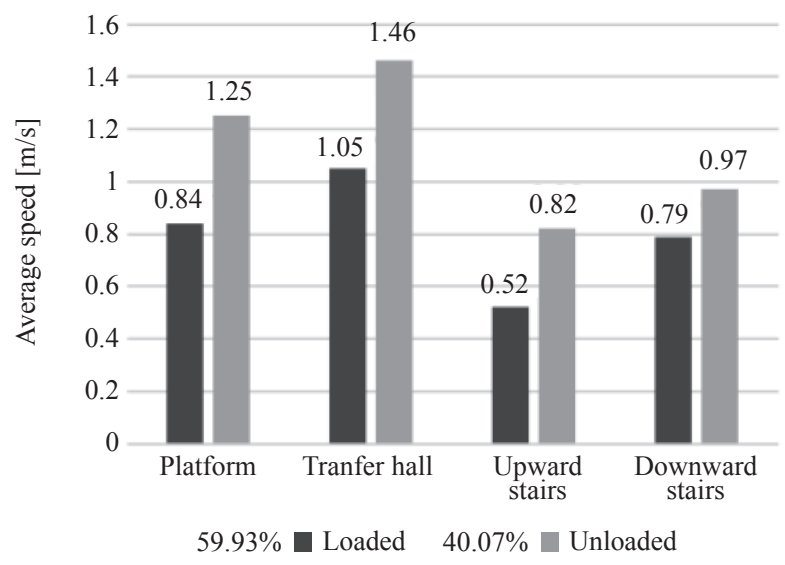

Figure 4-Average speed of different load condition

\subsubsection{Characteristics at ticket gates}

After observing the pedestrian behaviour at the ticket gates, the area near the ticket gate was divided into three parts: pace buffer area before the gate, passing area and the walking speed adjustment area, as shown in Figure 5. 


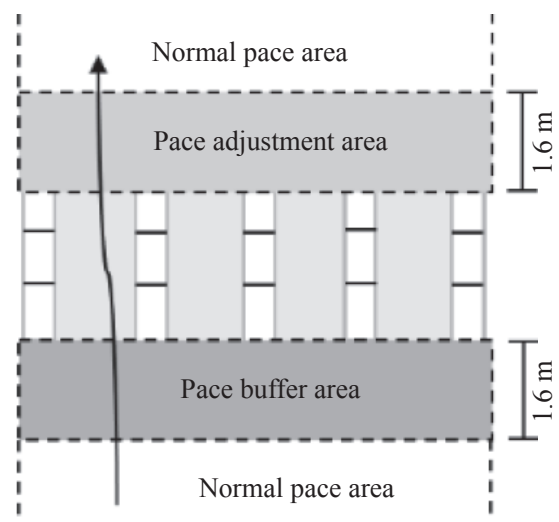

Figure 5-Area division at the ticket gates

As can be seen from Table 2, the pedestrians have different walking speeds in different parts of the gate area, but the difference between males and females is not significant (unlike that in other facilities). This may be attributed to the constraints from the limited width of the ticket gates and the surrounding pedestrians, which cause their speeds to converge.

Table 2 - Pedestrian expected speed

\begin{tabular}{||l|c|c||}
\hline Expected speed $[\mathrm{m} / \mathrm{s}]$ & Male & Female \\
\hline \hline Normal pace area & $1.25-1.50$ & $1.20-1.45$ \\
\hline Pace buffer area & $0.40-0.65$ & $0.40-0.65$ \\
\hline Passing area & $0.48-0.73$ & $0.48-0.73$ \\
\hline Pace adjustment area & $0.78-1.03$ & $0.73-0.98$ \\
\hline
\end{tabular}

\subsubsection{Characteristics of stairs/escalator selection behaviour}

In subway hubs, the escalators and stairs serve as the main facilities connecting the platform floor and the station floor, and the necessary equipment for pedestrian transfer. Generally speaking, pedestrians prefer to choose escalators when they coexist with the staircases, but this trend is subject to multiple factors.

The elevation of stairs and the number of queueing people

The pedestrian stairs/escalator selection behaviour was studied during the evening peak hours at three points: Exit $\mathrm{C}$ of JianGuoMen Station, Exit $\mathrm{B}$ of XiZhiMen Station and the exit of the platform floor of Beijing West Railway Station. The staircases at those points have different elevation heights.
The statistical results are shown in Figure 6. According to the results, the proportion of pedestrians who choose the stairs shows an increasing trend as the number of people waiting for the escalator increases, and the lower the elevation of the stairs, the more obvious is this trend.

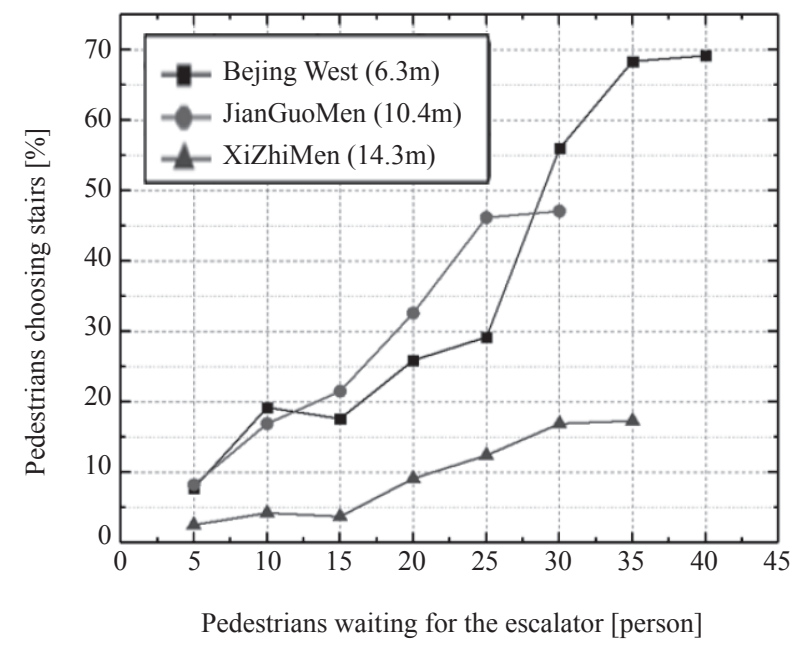

Figure 6-Selection results of different positions

\section{Load condition}

The pedestrian stairs/escalator selection behaviour at the exit of the platform floor of Beijing West Railway Station was observed from 05:00 to 05:30 p.m. and the statistical results based on the load condition are shown in Figure 7.

The probability of loaded pedestrians in the hub choosing escalators is $66.9 \%$, and the probability of unloaded pedestrians choosing escalators rises to $79 \%$. The result shows that the load condition has a great impact on the pedestrian stairs/escalator selection behaviour.

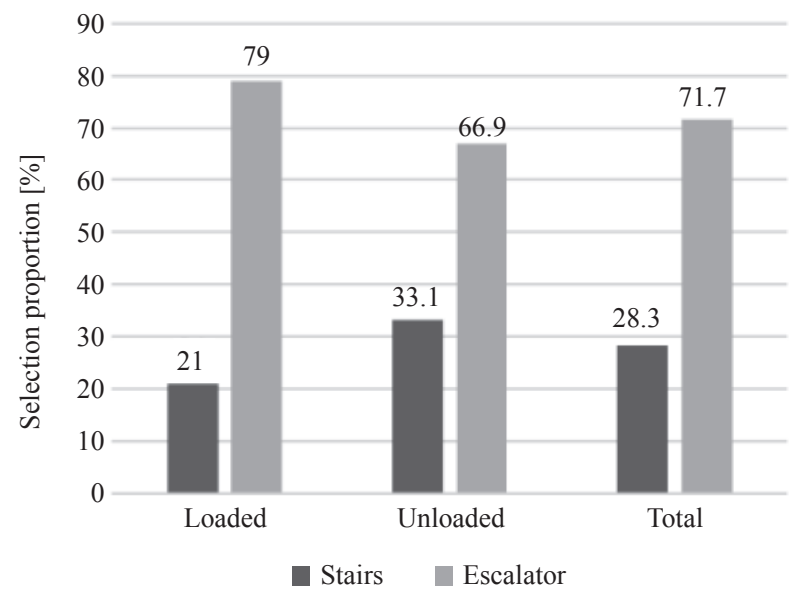

Figure 7 - Selection proportion of stairs and escalators based on load condition 


\subsection{Pedestrian flow lines}

The pedestrian flow lines of a traditional transfer channel are simple since all people move in the same direction, while in Beijing West Railway Station, the integral transfer hall replaces the transfer channel. The intersecting pedestrian flows of the east-west transfer passengers and the passengers entering and leaving the station result in complicated streamlines, as shown in Figure 8.

\section{THE SIMULATION MODEL OF PEDESTRIAN EVACUATION}

Aiming at analysing the evacuation process in the hub, a common add-on module in PTV Vissim - PTV Viswalk is used - for pedestrian microscopic simulation of Beijing West Railway Station. The pedestrian module in the software adopts Professor Helbing's social force model, in which the pedestrian's movement trajectory does not need to be defined in advance, and the pedestrian's movement is not restrained, so more reliable simulation results can be obtained.

\subsection{Construction of a social force model}

\subsubsection{Rationale}

In the social force model, social force is defined as the interaction between pedestrians and the environment, which can be described as follows:

$$
\begin{aligned}
& f_{a}(t)=m_{a} \frac{d v_{a}(t)}{d t}=f_{a}^{0}(t)+\sum_{i(i \neq a)} f_{a i}(t)+\sum_{w} f_{a w}(t)+ \\
& +\sum_{r} f_{a r}(t)+\xi_{n}(t)
\end{aligned}
$$

where:

$f_{a}^{0}(t)$ - self-driving force of pedestrian $a$;

$f_{a i}(t)$ - repulsive force from pedestrian $i$;

$f_{a w}(t)$ - repulsive force from obstacle $w$;

$f_{a r}(t)$ - attraction force from the environment;

$\xi_{n}(t)$ - disturbance term.
Generally, it is assumed that the pedestrian mass $m_{a}=m=1, \forall a$. When studying the social force model of non-specific situations, the attraction force from the environment is neglected, that is, $f_{a r}(t)$ in Equation 1 is omitted, and the following formula is obtained:

$$
f_{a}(t)=f_{a}^{0}(t)+\sum_{i(i \neq a)} f_{a i}(t)+\sum_{w} f_{a w}(t)+\xi_{n}(t)
$$

Set $\left\{S_{a}^{1}, \ldots, S_{a}^{k}, \ldots, S_{a}^{K}\right\}$ is defined as all the paths that "pedestrian $a$ " passes through during her/his travel, and $K$ is the total number of the paths. Then the terms on the right side of Equation 2 can be described as follows:

$$
f_{a}^{0}(t)=m_{a} \frac{V_{a}^{0} e_{a}(t)-V_{a}(t)}{\tau_{a}}
$$

where:

$V_{a} 0$ - expected speed of pedestrian $a$;

$e_{a}(t)-$ unit vector of $S_{a}{ }^{k}$ at time $t$; and

$e_{a}(t)=\frac{S_{a}^{K}-S_{a}(t)}{\left\|S_{a}^{K}-S_{a}(t)\right\|} ; \mathrm{V}_{a}(t)-$ actual speed of

pedestrian $a$ at time $t$;

$\tau_{a} \quad$ - slack time.

$$
f_{a i}(t)=m_{a} \frac{A_{a}}{B_{b}} \exp \left(-\frac{\left\|S_{a i}(t)\right\|}{B_{a}}\right) \frac{S_{a i}(t)}{\left\|S_{a i}(t)\right\|}
$$

where:

$S_{a i}(t)=S_{a}(t)-S_{i}(t)-$ position vector difference between pedestrian $a$ and $i$;

$A_{a}$ - strength of $f_{a i}(t)$;

$B_{b}-$ distance of $f_{a i}(t)$.

$$
f_{a w}(t)=m_{a} \frac{A_{w}}{B_{w}} \exp \left(-\frac{\left\|S_{a w}(t)\right\|}{B_{w}}\right) \frac{S_{a w}(t)}{\left\|S_{a w}(t)\right\|}
$$

where:

$S_{a w}(t)=S_{a}(t)-S_{w}^{a}(t)$-vector difference between the position of pedestrian $a$ and the position of the nearest point on obstacle $w$ from $a$;

$A_{w}$ - strength of $f_{a w}(t)$;

$B_{w}-$ distance of $f_{a w}(t)$.

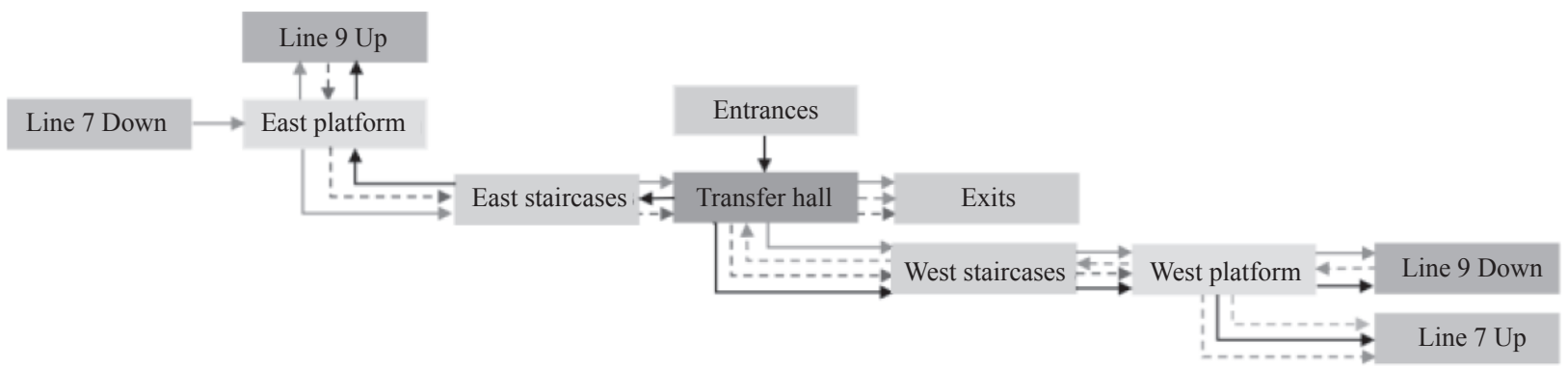

Figure 8 - Schematic diagram of pedestrian flow lines in Beijing West Railway Station 


\subsubsection{Parameter calibration}

The parameter of self-driving force. Considering a pedestrian as a circle, then the radius $r$ is the chest thickness or shoulder width of the human body. Referring to the previous study [10], we consider the static space requirements of pedestrians conforming to the uniform distribution of 0.4-0.6 metre.

Slack time. Slack time $\tau$ refers to the reaction time after a pedestrian is stimulated by the outside world. Related study [10] has shown that the reaction time for general external stimuli is about 0.1-0.5 s, and for more complex stimuli it is 1-3 s. Since the process of pedestrian speed adjustment is relatively simple, we suppose $\tau=0.5 \mathrm{~s}$.

Expected speed. The expected speed is the walking speed of a pedestrian without any restraints, and the pedestrians of different genders have different expected speeds. In the social force model, pedestrians will walk at their own expected speeds.

The pedestrians' walking speed is subject to multiple factors, which has been fully discussed in Section 2.2.1. The pedestrians' expected speed in the simulation model should follow a certain mathematical distribution type. We suppose the pedestrians' expected speed follows a uniform distribution within a certain range, which is shown in Table 1. The expected speeds of loaded pedestrians are reduced to $70 \%$ of the normal value. In addition, since the youths and the middle-aged make up $97 \%$ of the data sample and their speeds are close at various facilities, their speed differences have not been subdivided.

\subsection{Parameter calibration}

Before the simulation, the value of some default parameters of Viswalk need to be corrected according to the actual situation. In addition, some actual parameters need to be set while constructing the model. According to the analytical results above, the specific settings are as follows:
1) In the simulation, $56.73 \%$ male passengers and $43.27 \%$ female passengers are set. Their expected speeds have been set in the parameter calibration of the social force model.

2) The pedestrians are supposed to have the same walking speed at normal pace areas as the transfer hall, then the expected speeds of pedestrians at ticket gates follow the uniform distribution of the speed range shown in Table 2.

3) Forty percent of loaded pedestrians are set and their self-driving force parameter $r$ (the radius of a pedestrian) is set to 1.2 times that of unloaded pedestrians.

4) The proportions of pedestrians choosing escalators among loaded and unloaded ones are set to be $79 \%$ and $66.9 \%$, respectively.

5) Other parameters are kept at their default value.

\subsection{Evaluation indicators}

To evaluate the evacuation process in the hub and help identify the evacuation bottlenecks, the pedestrian density and the evacuation time have been chosen as evaluation indicators.

To directly reflect the impact of pedestrian density on the pedestrian evacuation, the 'level of service (LOS)' of pedestrian facilities is used to represent different evacuation situations of different pedestrian densities. Zhang Haili[11]'s revised classification standard of the LOS of pedestrian facilities is used according to her research in Beijing Subway, which is shown in Table 3.

According to the description of the division principle of LOS in Highway Capacity Manual [12] (HCM2000) of America, the pedestrians provided with A-level service can walk freely, whereas when LOS reaches $\mathrm{F}$, the pedestrians will be obviously limited and show a trend of queueing.

The evacuation time consists of three parts: the first is the time of entering the station, that is, the time taken by the pedestrian from the ticket gate to

Table 3 -Classification standard of Level of Service

\begin{tabular}{|c|c|c|c|}
\hline \hline LOS & Density (platform) & Density (channel) & Colour (CMYK) \\
\hline \hline A & $<0.66$ & $<0.26$ & $\square(255,0,0,255)$ \\
\hline B & $0.66 \sim 0.84$ & $0.26 \sim 0.47$ & $\square(255,0,255,255)$ \\
\hline C & $0.84 \sim 1.32$ & $0.47 \sim 0.73$ & $\square(255,0,255,0)$ \\
\hline D & $1.32 \sim 1.81$ & $0.73 \sim 1.19$ & $\square(255,255,255,0)$ \\
\hline E & $1.81 \sim 3.46$ & $1.19 \sim 1.89$ & $\square(255,255,128,0)$ \\
\hline F & $>3.46$ & $>1.89$ & $\square(255,255,0,0)$ \\
\hline
\end{tabular}


Chen Q, Bai Y, Zheng N. Simulation and Optimization of Pedestrian Regular Evacuation in Comprehensive Rail Transit Hub...

Table 4 - Statistics of evacuation time

\begin{tabular}{||c|c|c|c|c||}
\hline \multicolumn{2}{|c|}{ Origin-destination } & ST & AT & Relative error \\
\hline \hline O & D & - & 142 & $5.8 \%$ \\
\hline East & L9 Down & 134.26 & 125 & $3.1 \%$ \\
\hline East & L7 Up & 121.21 & 84 & $2.0 \%$ \\
\hline East & L9 Up & 82.37 & 102 & $4.1 \%$ \\
\hline West & L9 Down & 97.99 & 95 & $3.2 \%$ \\
\hline West & L7 Up & 92.08 & 132 & $0.8 \%$ \\
\hline West & L9 Up & 130.93 & 19 & $0.5 \%$ \\
\hline L9 Down & L7 Up & 18.91 & 166 & $4.0 \%$ \\
\hline L9 Up & L7 Up & 159.57 & 155 & $0.2 \%$ \\
\hline L7 Down & L9 Down & 154.73 & 23 & $3.2 \%$ \\
\hline L7 Down & L9 Up & 22.28 & 83 & $1.2 \%$ \\
\hline L9 Down & North & 82.02 & 106 & $1.9 \%$ \\
\hline L9 Down & South & 104.02 & 90 & $4.6 \%$ \\
\hline L9 Up & North & 86.00 & 135 & $2.3 \%$ \\
\hline L9 Up & South & 131.96 & 104 & $1.7 \%$ \\
\hline L7 Down & North & 102.24 & 143 & $4.3 \%$ \\
\hline L7 Down & South & 137.09 & & \\
\hline
\end{tabular}

the platform; the second is the time of leaving the station, the evacuation time taken from the platform to the exit; the third is the transfer time, that is, the time taken for transferring from one train to another.

\subsection{Model suitability verification}

To verify the suitability of the model, the simulated pedestrian evacuation time is compared with the real data before formal simulation.

Twenty pedestrians of each travel type were tracked, and their average evacuation time was taken as the actual time (AT). As the simulation goes, the pedestrian evacuation time will increase as more people enter the hub and eventually reach a stable value. Therefore, the data from $600 \mathrm{~s}$ to $960 \mathrm{~s}$ were chosen, and the average evacuation time during this period was taken as simulated evacuation time (ST). The comparison results are shown in Table 4.

The simulation results are basically consistent with the actual results: the maximum relative error is $5.8 \%$ and the average relative error is $2.7 \%$. Therefore, the simulation model can reflect the actual situation with high credibility.

\section{SIMULATION RESULT ANALYSIS AND OPTIMIZATION}

During the evening peak, the departure intervals of Line 9 and Line 7 are $195 \mathrm{~s}$ and $240 \mathrm{~s}$, respectively. We have adopted the actual train arrival sequence and interval during evening peak hours in the simulation process: the up train of Line 9 arrives first, the down train of Line 9 arrives 30 s later, the down train of Line 7 arrives $90 \mathrm{~s}$ later, and the up train of Line 7 arrives $120 \mathrm{~s}$ later.

According to the real data obtained from the Beijing Subway, during the evening peak hours, the inbound passenger flow of Beijing West Station peaked at 7,710 persons/hour during the period from 5 p.m. to 6 p.m.. Thus, in order to analyse the pedestrian evacuation process during that period, the inbound passenger flow of 7,710 persons/hour is evenly assigned to the entrances on the west and east sides of the transfer hall in the simulation, and the running time of the simulation is set at 3,600 seconds.

\subsection{Platform floor}

\subsubsection{Analysis of pedestrian density distribution}

The passengers from Subway Line 7 and Line 9 gather at the platform floor. It is chosen to analyse the simulation process of the first $300 \mathrm{~s}$, and the density distribution of pedestrian flow is shown in Table 5.

Judging from the density distribution maps, the evacuation process during the evening peak presents periodic changes. The pedestrian density of the platform floor maintains a high level for most of the time, which is likely to cause congestion. 
Chen Q, Bai Y, Zheng N. Simulation and Optimization of Pedestrian Regular Evacuation in Comprehensive Rail Transit Hub...

Table 5 - Density distribution of pedestrian flow changing with simulation time (platform floor)

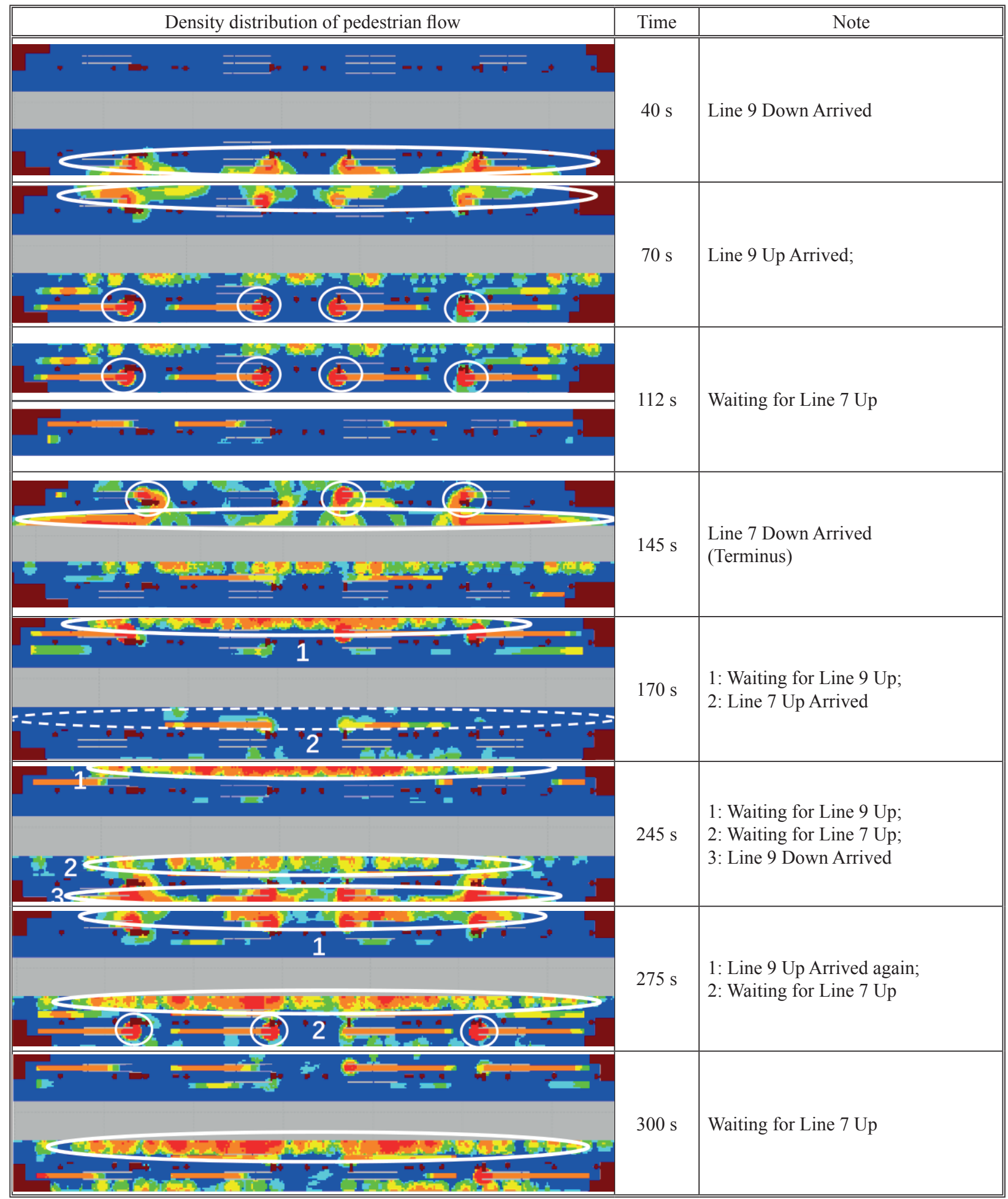

High-density areas (circled in the maps) emerge frequently at the stairs and the waiting areas, which requires a long time to evacuate. Each train can basically accommodate all waiting passengers and empty the platform, and will not let the passengers stranded. The simulation results are consistent with the actual observations to a large extent.
In addition, it has been found that the same-platform transfer is convenient: it takes only a small amount of time to transfer passengers from one side to the other side of the platform. However, there are certain disadvantages. First, the platform plays part of the role of transfer channel and assumes greater traffic pressure, which requires better 
evacuation capacity of the transfer platform. Second, the arrival time of the two trains which transfer at the same platform also has a greater impact on the pedestrian evacuation. When two trains arrive at the same time, the pedestrians' movement will be seriously hindered by each other, which may cause their evacuation time to be greater than the total time when the two trains arrive alone.

\subsubsection{Identification and optimization of the evacuation bottleneck}

According to the actual situation and simulation results, it is found that the pedestrian-intensive areas of the platform floor were mainly concentrated near the staircases, and the maximum risk point is shown in Figure 9.

Generally speaking, there are three ways to mitigate the traffic pressure of the aforementioned risk areas: first, slow the pedestrians' walking speed by changing their flow lines, such as adding a diversion bar or buffer column; second, improve the capacity of equipment and facilities in the risk area, such as increasing the number of ticket gates, the width of the passengers and so on; third, reduce the passenger flow volume entering the risk area per unit time, such as taking current limiting measures or adjusting the departure interval of the trains.

According to our field investigation, the equipment allocation of the platform floor has approached saturation. Although the distance between the escalator and the stairs is nearly $2 \mathrm{~m}$, there is a load-bearing column arranged between them, and the outer rim of the stairs is only 2.35 $\mathrm{m}$ away from the platform screen doors. Therefore, there is no space to improve the capacity of facilities, so we can only consider to shorten the departure interval or to take the current limiting measures to alleviate the congestion.

During the evening peak, the departure interval of Line 9 is $3 \min 15 \mathrm{~s}$, and the departure interval of Line 7 is 4 min, which shows a large adjustment space. Therefore, referring to the recent interval change from $3 \mathrm{~min} 15 \mathrm{~s}$ to $3 \mathrm{~min} 01 \mathrm{~s}$ of Line 9 during the morning peak, the interval of Line 9 is adjusted during the evening peak of the simulation model to $3 \mathrm{~min} 01 \mathrm{~s}$ as well, and the interval of Line 7 should be $3 \min 30 \mathrm{~s}$. The result of the optimized simulation helps evaluate the optimization effect.

We extracted the original and optimized the pedestrian simulation data at the maximum risk point by 1 hour at the interval of $30 \mathrm{~s}$. Then we compared their pedestrian density timing changes and the simulation time ratio at different levels of service, as shown in Figure 10.

The pedestrian density at the bottleneck changes periodically with the arrival time of the trains. Before optimization, the maximum pedestrian density reached 5.97 person $/ \mathrm{m}^{2}$, and the LOS of the bottleneck was below $\mathrm{C}$ level during $63.3 \%$ of the simulation time, which indicates a great possibility of congestion. In the simulation process, to make the LOS reach $\mathrm{C}$ or above, the maximum time required is $218 \mathrm{~s}$, and the minimum time required is at least $100 \mathrm{~s}$. To conclude, the evacuation capacity of the platform can meet the demand of pedestrian flow during the evening 5:00 s, but the LOS is poor.

After the departure intervals are reduced, the maximum pedestrian density at the risk point was significantly reduced to 3.21 person $/ \mathrm{m}^{2}$, and the average pedestrian density was also reduced from 1.68 person $/ \mathrm{m}^{2}$ to 1.04 person $/ \mathrm{m}^{2}$. Furthermore, the LOS after optimization has also been significantly improved, which indicates that the congestion has been mitigated effectively. The maximum time for improving the LOS above $\mathrm{C}\left(<0.84\right.$ person $\left./ \mathrm{m}^{2}\right)$ is shortened from $218 \mathrm{~s}$ to $166 \mathrm{~s}$, and the minimum time is shortened from $100 \mathrm{~s}$ to $67 \mathrm{~s}$. The comparison results above verify the effectiveness of the optimization measure.

\subsection{Transfer hall}

\subsubsection{Analysis of pedestrian density distribution}

The station hall of Beijing West Railway Station is an integrated transfer hall, which concentrates the inbound, outbound and transfer passengers. The

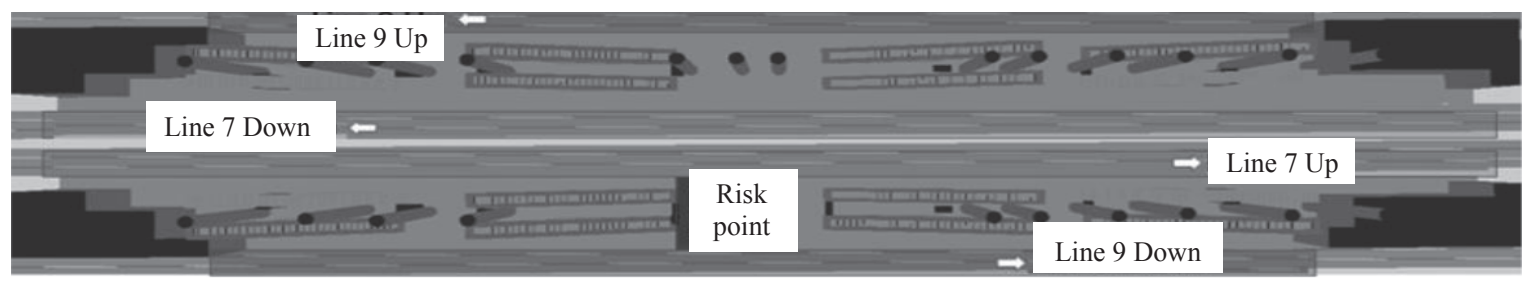

Figure 9 - Location of the maximum risk point at the platform floor 


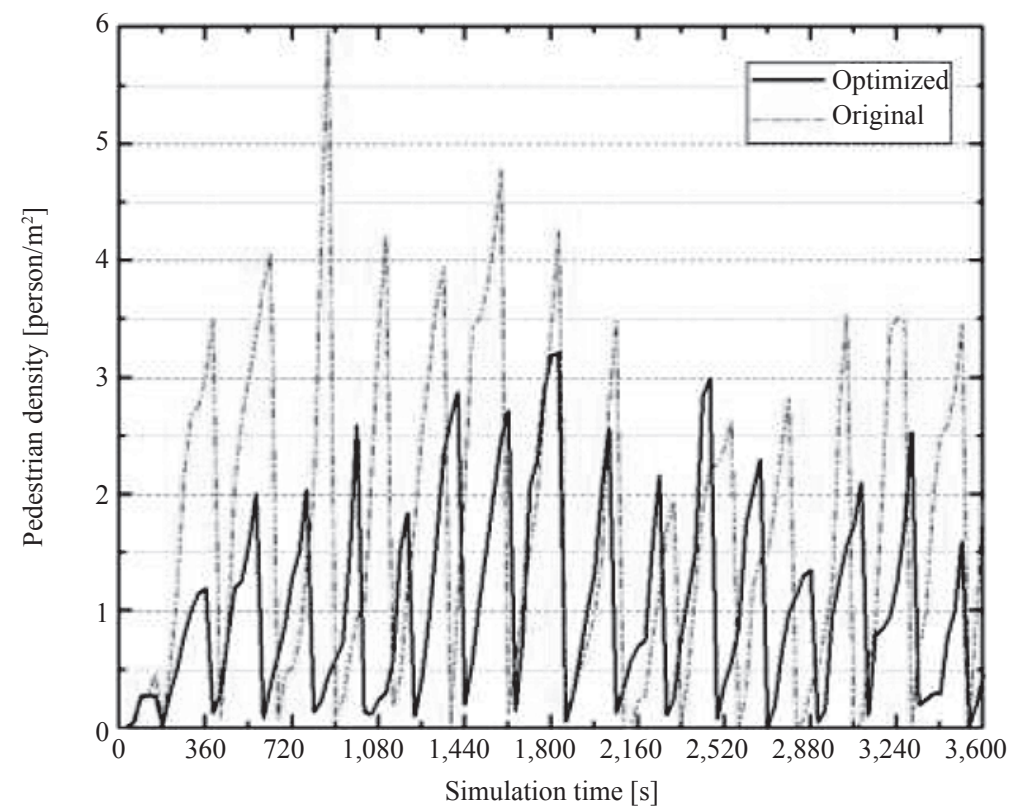

a) Pedestrian density changes with time

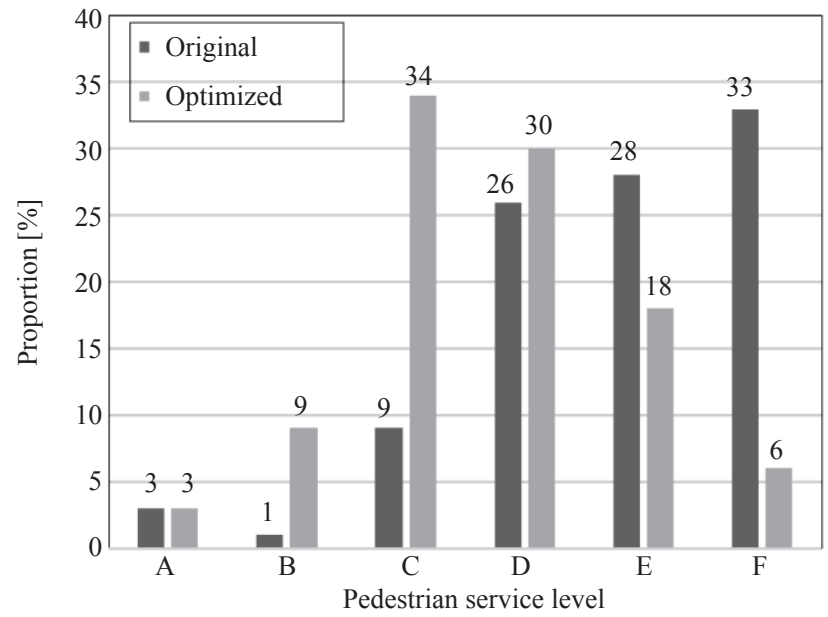

b) Pedestrian density allocated in proportion

Figure 10 - Comparison of pedestrian density of the risk point at the platform floor

passenger flow distribution changes with time periodically, so we choose to analyse the simulation process in one period (from $700 \mathrm{~s}$ to $880 \mathrm{~s}$ ) as shown in Table 6.

Compared with the platform floor, the pedestrian density at the transfer hall was generally lower, and there emerged only a few high-density areas (circled in the maps), which fully shows the advantages of evacuating pedestrians effectively from the integrated transfer hall.

The transfer pedestrian flow and the outbound pedestrian flow show cyclical fluctuations and they can be evacuated effectively in a short period of time, while the inbound pedestrian flow is constant without respite, forming unremitting high-density queueing areas at the staircases. To conclude, the transfer hall can accommodate all kinds of pedestrians during the evening peak, and the pedestrians can be evacuated to their respective target directions without causing severe congestion. The simulation results are basically consistent with the actual observations.

\subsubsection{Identification and optimization of the evacuation bottlenecks}

Based on the field research and simulation results, three high-density pedestrian areas in the transfer hall have been identified, and they are shown in Figure 11.

The causes of the three risk points are different: the first one is caused by the vertical interweaving flow lines of outbound passengers and transfer passengers; the second one at the staircase is due to the 
Table 6 - Density distribution of pedestrian flow changing with simulation time (transfer hall)

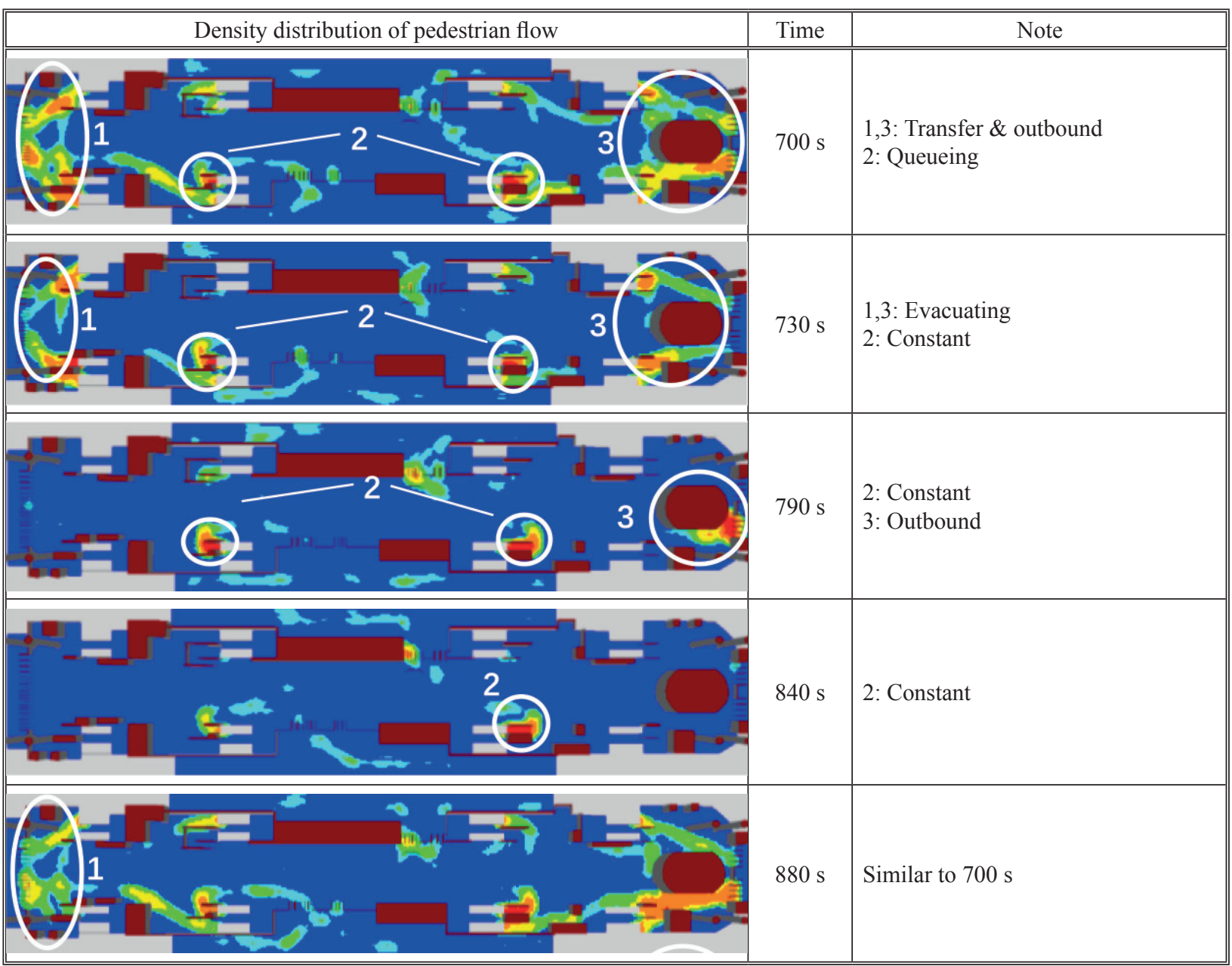

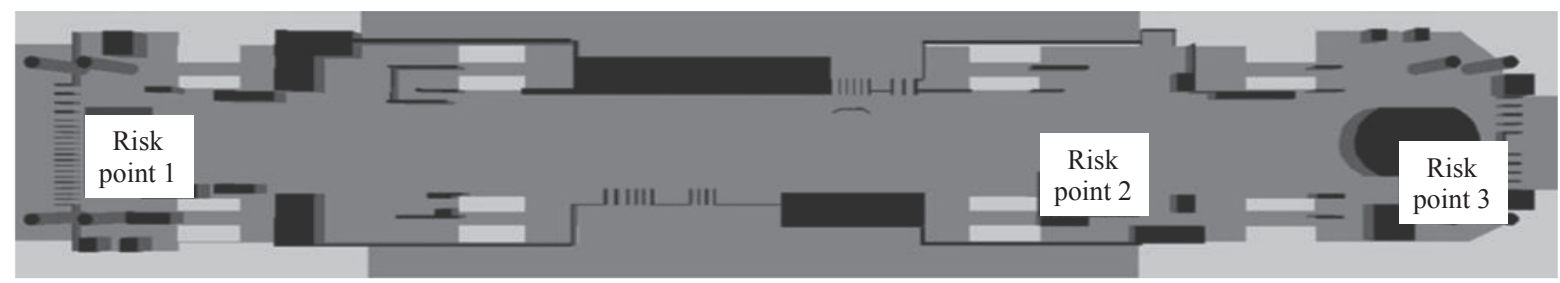

Figure 11 - Location of the maximum risk points in transfer hall

queueing group stemming from the large number of inbound passengers; the last one on the south side is caused by the limited number of ticket gates and the restraint of the dispatching room.

\section{Optimization of the maximum risk point}

According to the actual situation and simulation results, the maximum risk point of the transfer hall is the queuing area at the staircase (Risk Point 2 in Figures 5-8). Since the area near the staircase is relatively spacious, a 3.5-metre-long diversion bar at the risk point is recommended to change the pedestrian flow lines and speed, and to improve the situation of disorderly queuing and congestion.
After having applied the optimization measure to the simulation model, we ran the optimized model again. Then we extracted the original and optimized pedestrian simulation data at the maximum risk point for one hour at the interval of $30 \mathrm{~s}$. The comparison results of their pedestrian density timing changes and the simulation time ratio at different levels of service are shown in Figure 12.

Before the optimization, the maximum pedestrian density at the risk point was 2.88 person $/ \mathrm{m}^{2}$. Within $87 \%$ of the time, the LOS of the area was in D level and below. In addition, the pedestrian density at the staircase no longer dropped to zero after a period of simulation, and the lowest value was 


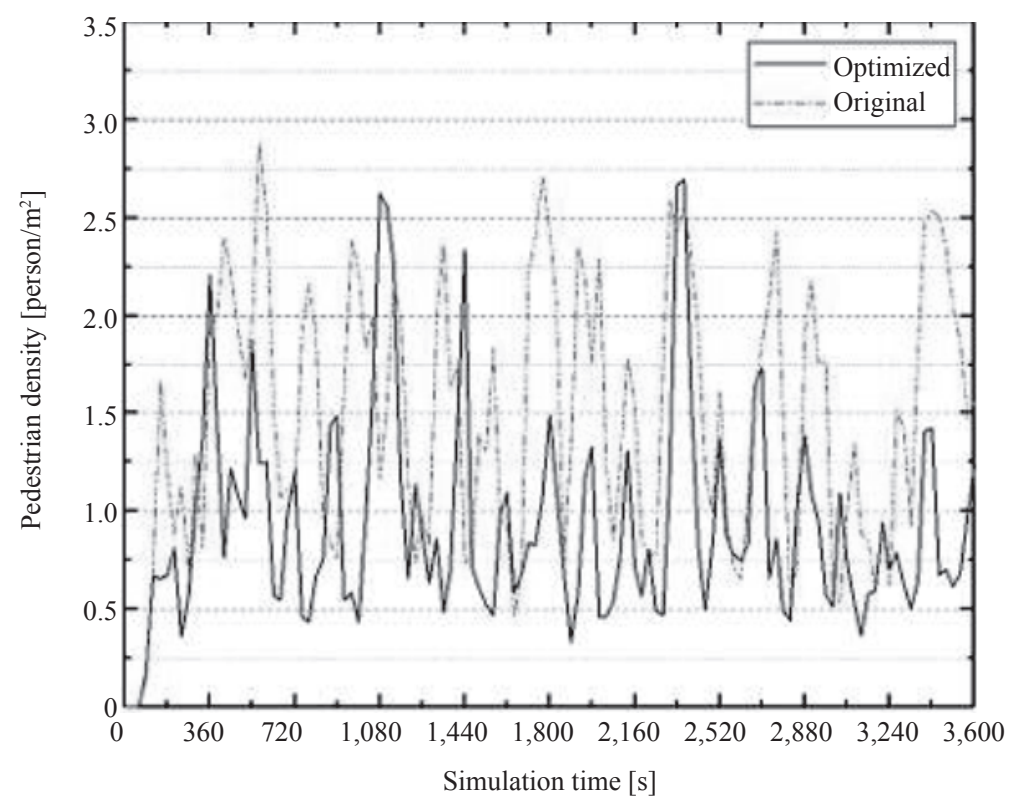

a) Pedestrian density changes with time

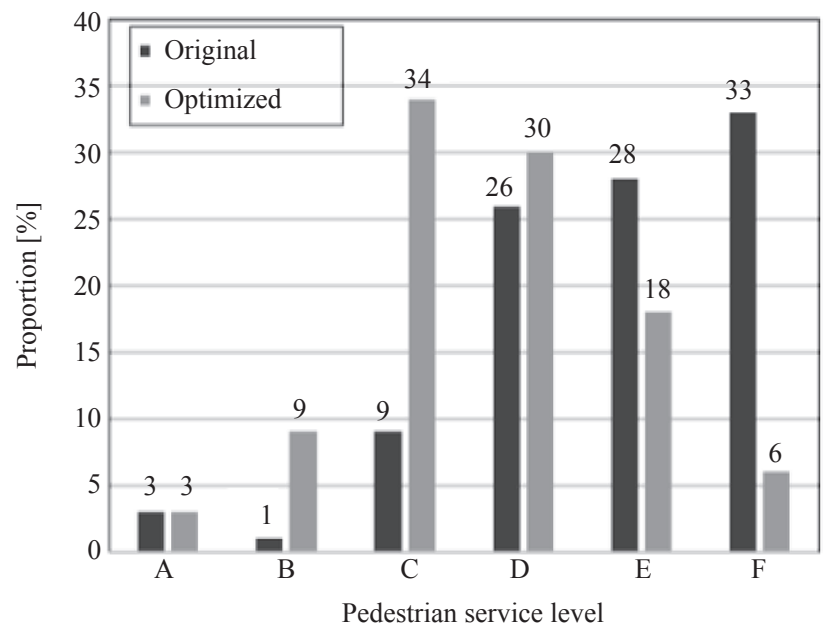

b) Pedestrian density allocated in proportion

Figure 12 - Comparison of pedestrian density of the risk point at the transfer hall

around 0.5 person $/ \mathrm{m}^{2}$. According to the research video, it is found that this risk point is adjacent to the entrance, and the platform connected by the stairs attracts passengers to the up train of Line 7 as well as the down train of Line 9, so constant passenger flow from the entrance would wait here for the escalator to get down.

It can be seen from Figure $12 a$ that after setting the diversion bar at the stairway, the maximum pedestrian density at the risk point is significantly reduced, and the average pedestrian value is also reduced from 1.48 person $/ \mathrm{m}^{2}$ to 0.95 person $/ \mathrm{m}^{2}$. Figure $12 b$ shows that the LOS has been significantly improved, which indicates that the congestion at the risk point has been effectively mitigated. Therefore, the effectiveness of the optimization is verified.
In summary, it can be considered that the optimization measures of setting a diversion fence is effective in optimizing the evacuation of pedestrians at the stairway of the station hall.

\section{Optimization of the risk point at the south exit}

The emergence of this risk point is due to the dispatching room which occupies a large part of the space near the south exit and the insufficient number of ticket gates. Therefore, the pedestrians' walking area is compressed. However, due to the compression of the space, the passengers have formed an orderly pedestrian flow before reaching the exit, which help them avoid hindering each other and disorderly path-finding behaviour, and make the congestion time not that long. 


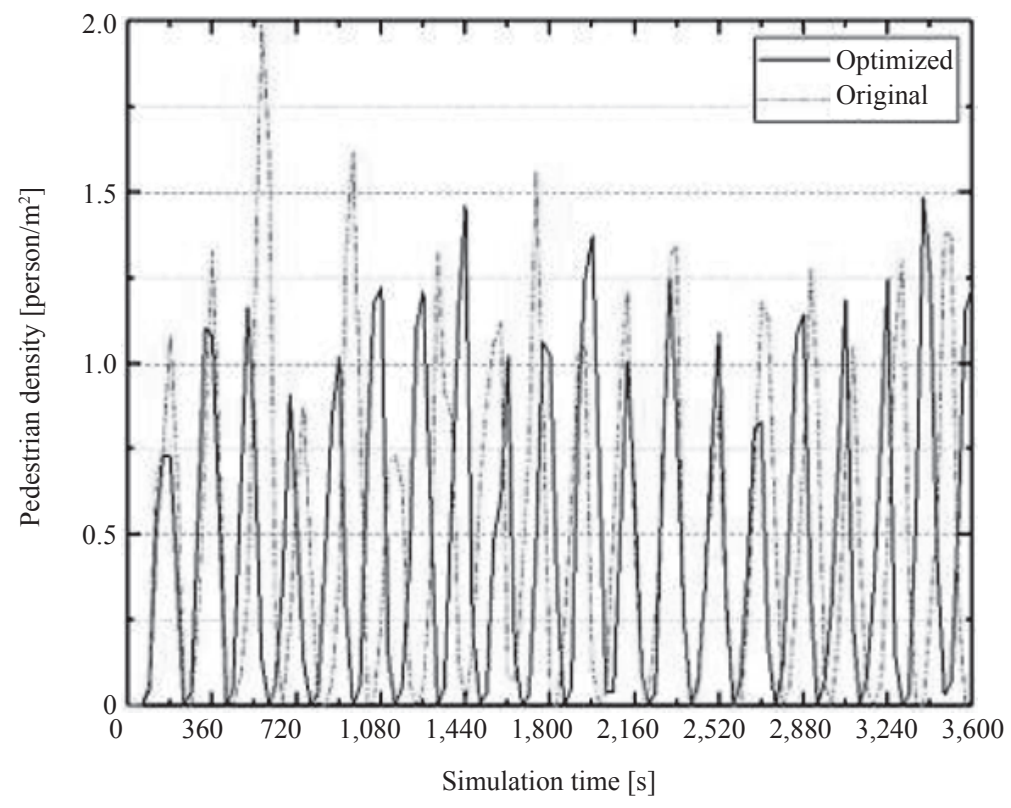

a) Pedestrian density changes with time

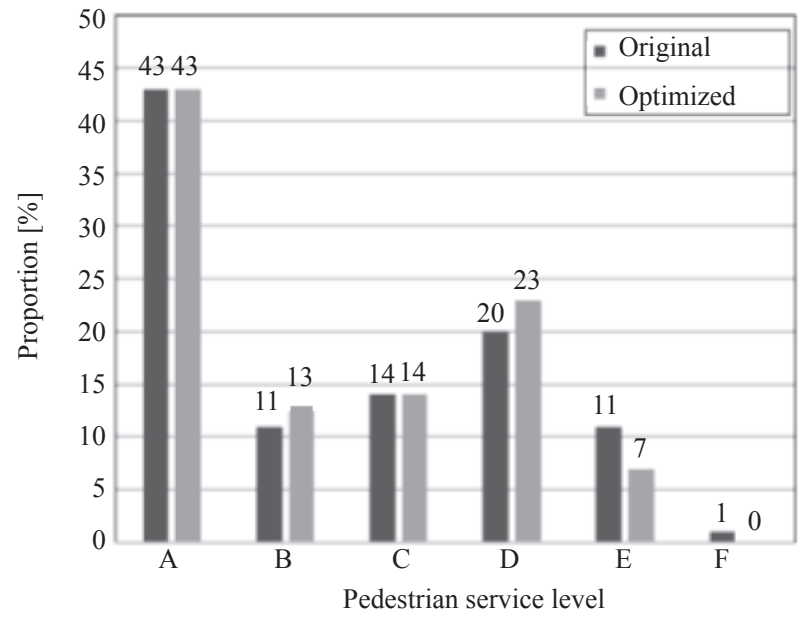

b) Pedestrian density allocated in proportion

Figure 13 - Comparison of pedestrian density of the risk point at south exit

Since there is enough spare space at the south exit, we propose to add three ticket gates to improve the passing capacity of the facilities at the south exit. After adjusting the simulation model, we extract the simulation data of one hour at the risk point and the comparison results are shown in Figure 13.

Before the optimization, the maximum pedestrian density of this area during the simulation was 1.99 person $/ \mathrm{m}^{2}$. In $54 \%$ of the simulation time, the LOS of this area kept above $\mathrm{C}$ level, which indicates that this area showed no signs of congestion in more than half of the simulation time. In addition, evacuating the pedestrians in this area to $\mathrm{C}$ level above only required approximately 100 seconds.
However, the optimization result (especially for LOS) does not show remarkable improvement. Therefore, the main cause of the congestion at the South exit might be the dispatching room adjacent to the South exit. We removed the dispatching room and increased the number of ticket gates at the same time, then ran the simulation model again. The comparison of the simulation results is shown in Figure 14.

Results show that the optimization measure greatly reduced the pedestrian density at the South exit, and the maximum density decreased from 1.99 person $/ \mathrm{m}^{2}$ to 1.07 person $/ \mathrm{m}^{2}$. In the simulation process, to make the LOS reach $\mathrm{C}$ or above, the average time required is shortened from $100 \mathrm{~s}$ to $43 \mathrm{~s}$. The 


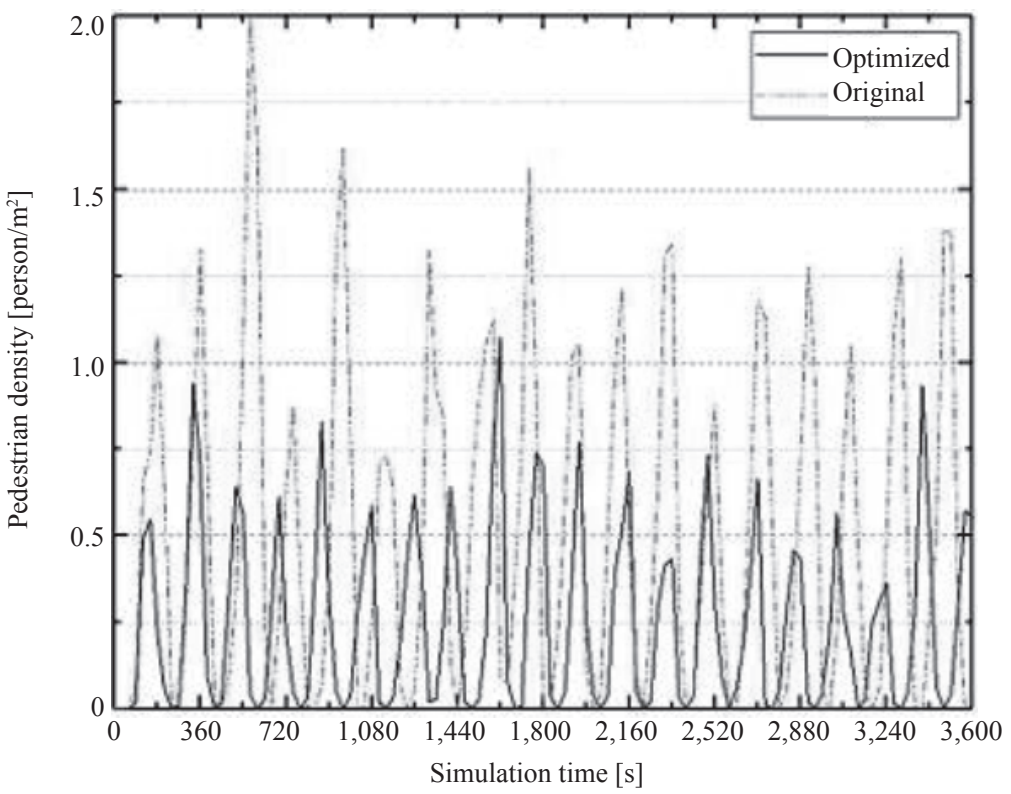

a) Pedestrian density changes with time

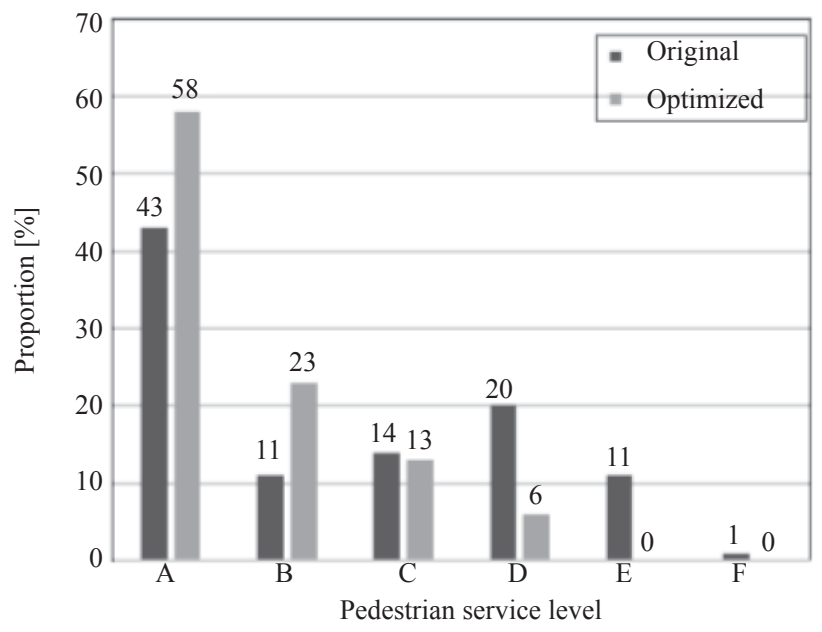

b) Pedestrian density allocated in proportion

Figure 14 - Comparison of pedestrian density of the risk point at the south (second optimization)

LOS has also been improved significantly, which indicates that the congestion has been mitigated effectively. In a nutshell, it can be verified that the optimization measure of adding ticket gates and removing the dispatching room is helping in releasing the congestion at the South exit.

\section{CONCLUSION}

It is of great significance to study the characteristics of pedestrian evacuation in a comprehensive rail transit hub and to propose further optimization measures, as the bottlenecks in the hub have become a constraint on pedestrian evacuation. Taking Beijing West Railway Station as the research object, this paper constructed an effective simulation model of pedestrian evacuation which can well represent the pedestrian characteristics in the hub. The model is capable of visually displaying real evacuation process, and all of relative data such as pedestrian density and evacuation time can be extracted, helping us to identify the evacuation bottlenecks. After proposing plausible optimization measures, the simulation model can also be used to verify the effectiveness of the measures by comparing the simulation results.

According to the simulation results, we found a periodical change of pedestrian density from both the platform floor and the transfer hall. In addition, the pedestrian density of the transfer hall was generally lower than that of the platform floor. The location and the causes of the risk points were also different. Therefore, the optimization measures for each risk point had to be proposed according to the 
specific situation. The comparison of the original and optimized simulation results showed that the measures we proposed can mitigate the congestion at the risk points effectively.

陈翅楚，白雁，郑楠

\section{轨道交通综合枢纽站行人疏散仿真优化研 究——以北京西站为例}

\section{摘要}

随着城市轨道交通拥堵问题的日益增多，有关 城市轨道交通系统的行人疏散研究得到了广泛的开 展。然而, 对城市综合轨道交通枢纽的研究较少。 本文以涵盖城市轨道交通和城际轨道交通的综合换 乘枢纽北京西站作为研究对象，首先分析了行人疏 散特征，然后在PTV Viswalk中建立了基于社会力的 北京西站仿真模型。将该模型应用于真直观显示实 际疏散过程, 帮助识别疏散瓶颈。结果表明, 不同 设施的风险点具有不同的成因和特点。此外，改变 该仿真模型的一些参数, 就可以将其用于评估不同 优化措施的有效性。

\section{关键词}

轨道交通, 综合换乘枢纽, 行人疏散

仿真, PTV Viswalk, 优化措施

\section{REFERENCES}

[1] Li J, Zhang JH. [Path-based network flow control emergency evacuation model and algorithm]. Journal of Natural Disasters. 2012;(6): 9-18.

[2] Yu WJ, Chen R, Dong LY, et al. Centrifugal force model for pedestrian dynamics. Physical Review E. 2005;72(2): 026-032.
[3] Xu Y, Huang HJ. Simulation of exit choosing in pedestrian evacuation with consideration of the direction visual field. Physical A: Statistical Mechanics and its Applications. 2012;391(4): 991-1000.

[4] Guo RY, Huang HJ. Theoretical analysis and simulation of pedestrian evacuation under invisible conditions. Simulation. 2012;88(9): 1138-1148.

[5] Guo RY, Huang HJ, Wong SC. Route Choice in Pedestrian Evacuation under Conditions of Good and Zero Visibility: Experimental and Simulation Results. Transportation Research Part B: Methodological. 2012;46(6): 669-686.

[6] Yue H, Hao HR, Chen XM, et al. Simulation of pedestrian flow on square lattice based on cellular automata model. Physica A: Statistical Mechanics and its Applications. 2007;384(2): 567-588.

[7] Muramatsu M, Irie T, Nagatani T. Jamming transition in pedestrian counter flow. Physica A: Statistical Mechanics \& Its Applications. 1999;267(S3-4): 487-498.

[8] Muramatsu M, Nagatani T. Jamming transition in two-dimensional pedestrian traffic. Physica A: Statistical Mechanics \& Its Applications. 2000;275(1-2): 281-291.

[9] Helbing D, Molnár P. Social force model for pedestrian dynamics. Physical Review E Statistical Physics Plasmas Fluids \& Related Interdisciplinary Topics. 1995;51(5): 4282.

[10] Hoogendoorn SP, Daamen W. Microscopic calibration and validation of pedestrian models: Cross-comparison of models using experimental data. In: Schadschneider A, Pöschel T, Kühne R, Schreckenberg M, Wolf DE. (eds.) Traffic and Granular Flow'05. Berlin, Heidelberg: Springer; 2005. p. 329-340.

[11] Zhang HL. [Study on the Simulation and Evaluation of Passenger Access Levels for Service in MTR Station]. Graduate thesis. Beijing Jiaotong University; 2011.

[12] National Research Council (NRC). Highway Capacity Manual 2000. Washington, D.C; 2000. 